\title{
Respuesta y riesgos de los antidepresivos durante la infancia y la adolescencia
}

\author{
Response and risk of antidepressant treatment during childhood and adolescense
}

\section{Objetivo}

Evaluar la eficacia y el riesgo de ideación e intentos suicida asociado a la utilización de antidepresivos en pacientes con trastorno depresivo mayor (TDM) obsesivo compulsivo (TOC) y de ansiedad no TOC (TAnoTOC) en niños y adolescentes.

\section{Fuente y selección de estudios}

PubMed (1988 a 2006) reportes de agencias reguladoras estadounidenses y británicas, resúmenes de encuentros científicos (1998 a 2006) registros de estudios clínicos e información de los autores. Se seleccionaron estudios publicados y no publicados aleatorizados, controlados con placebo o grupo paralelo que hubieran usado antidepresivos de segunda generación como inhibidores selectivos de la recaptación de serotonina (ISRS) nefazodona, venlafaxina y mirtazapina, en menores de 19 años.

\section{Extracción de datos}

Características del estudio, resultados de eficacia y reportes espontáneos de ideación suicida/intento de suicidio.

\section{Resultados principales}

Fueron identificados 27 estudios pediátricos, 15 de TDM, seis de TOC y seis de TAnoTOC. Los resultados fueron estimados por métodos de efecto aleatorio* y fueron favorables para TDM $(11 \%$; IC95\% 7,1 a 14,9$)$ TOC $(19,8 \%$; IC95\% 13 a 26$)$ y
TAnoTOC (37,1\%; IC9\% 22,5 a 51,7) correspondiendo a un número necesario para tratar (NNT) de 10 (IC95\% 7 a 15) 6 (4 a 8) y 3 (2 a 5) respectivamente.

Se evidenció un aumento estadísticamente no significativo en el riesgo de ideación e intento suicida pero no hubo suicidios consumados. El análisis estratificado por edad mostró que para los niños menores de 12 años con TDM sólo fluoxetina mostró beneficios sobre el placebo, habiéndose evidenciado una menor eficacia global de los tratamientos para TDM a edades más tempranas y a mayor duración de la depresión.

\section{Conclusiones}

Los antidepresivos fueron eficaces en pediatría para el tratamiento de TDM, TOC y TAnoTOC, aunque los efectos fueron más importantes en los TAnoTOC, intermedios en TOC y más modestos en TDM. Sólo fluoxetina demostró beneficios con respecto al placebo en menores de 12 años con TDM. Los beneficios de los antidepresivos parecen ser considerablemente mayores que los riesgos de ideación o intento suicida. La relación entre los riesgos y los beneficios varió en función de la indicación, la edad, la cronicidad y las condiciones de cada estudio.

Palabras claves: tratamiento antidepresivo, ideación suicida, intento de suicidio, niños, adolescentes.

Key words: antidepressant treatment, suicidal ideation, suicide attempts, pediatrics, adolescents.

Fuente de financiamiento: National Institute of Mental Health y National Institutes of Health (Estados Unidos) y Robert Wood Johnson Foundation

\section{Comentario}

El presente meta-análisis se realizó en el contexto de la polémica desencadenada por las advertencias que ordenó durante 2004la Administración de Alimentos y Drogas de Estados Unidos (FDA) referente al aumento de la conducta e ideación suicida con el uso de antidepresivos en niños y adolescentes ${ }^{1}$ Consistentemente con el análisis realizado por la FDA, el presente trabajo describe un aumento del riesgo de ideación y/o intento suicida asociado con el tratamiento antidepresivo menor al 1\% (en aquel había resultado de 1,7\%). Los autores atribuyen la diferencia a la técnica usada para combinar los estudios y a que agregaron siete estudios no incluidos en el anterior análisis, arrojando resultados similares otras revisiones sobre el tema ${ }^{2,3,4}$. Por otro lado, algunos trabajos cuestionan que el aumento de la ideación y/o el intento suicida derive realmente en un aumento de suicidios consumados, refiriendo que el número de estos últimos disminuyó drásticamente desde la aparición de los ISRS ${ }^{5}$ y que la depresión en sí sería el mayor factor de riesgo aislado de suicidio adolescente ${ }^{6}$ por lo que si mejora el cuadro, la probabilidad final de suicidio sería menor. Sin embargo, no se consideró que otros efectos adversos no reportados como activación, insomnio, desinhibi-

ción, manía, irritabilidad o agitación puedan precipitar la ideación o los intentos suicida ${ }^{7}$. Vale la pena destacar que según este trabajo, para el caso del TDM pediátrico, la respuesta a antidepresivos es solo un $11 \%$ más frecuente que la respuesta a placebo (61 vs. $50 \%$ ) debiendo ser tratados diez pacientes para que uno responda adicionalemente a la mejoría esperada con el placebo. A su vez, un paciente cada 112 tratados tendría mayor riesgo de aumento de la ideación o conducta suicida que con placebo.

\section{Consclusiones del comentador}

Estos resultados apoyan un uso cuidadoso y estrechamente monitoreado de los antidepresivos para todas sus indicaciones pero, sobre todo, la elección conjunta del tratamiento con el paciente y la familia, luego de una discusión colaborativa en la que se les informe de los potenciales beneficios y riesgos de estas medicaciones, en comparación con tratamientos no farmacológicos e inclusive con ausencia de tratamiento.

Ver glosario*

Sebastián Cukier [ Psiquiatra, Hospital Tobar García y Fundación para la Lucha de las Enfermedades Neurológicas de la Infancia, Escobar. sebastiancukier@ hotmail.com ]

Recibido el 02/04/08 y aceptado el 24/05/08.

Cukier S. Utilidades y riesgos de los antidepresivos durante la infancia y la adolescencia. Evid. actual. práct. ambul; 11(3):70, May-Jun 2008. Comentado de: Bridge J y col. Clinical Response and Risk for Reported Suicidal Ideation and Suicide Attempts in Pediatric Antidepressant Treatment. A Meta-Analysis of Randomized Controlled Trials. JAMA 2007 Apr 18. 297 (15):1683-1696. PMID: 17440145. Disponible en URL: http://jama.ama-assn.org/cgi/reprint/297/15/1683

Referencia

1. US Food and Drug Administration. Relationship between psychotropic drugs and pediatric suicidality: review and evaluation of clinical data. http://www.fda.gov/ohrms/dockets/ac/04/briefing/2004-4065b1-10-TAB08-Hammads-Review.pdf. (último acceso 02/04/08).

2. Cheung A y col. Review of the efficacy and safety of antidepressants in youth depression. J Chile Psicol Psychiatry. 2005;46:735-754

3. Hammad T y col. Suicidality in pediatric patients treated with antidepressant drugs. Arch Gen Psychiatry. 2006;63:332-9.

4. Kaizar E y col. Do antidepressants cause suicidality in children? A Bayesian meta-analysis. Clin Trials. 2006;3:73-98.

4. Kaizar E y col. Do antidepressants cause suicidality in children? A Bayesian meta-analysis. Clin Trials. 2006;3:73-98.
5. Olfson M y col. Relationship between antidepressant medication treatment and suicide in adolescents. Arch Gen Psychiatry 2003;60:978-82.

5. Olfson M y col. Relationship between antidepressant medication treatment and suicide in adolescents. Arch Gen Psychiatry 2003;60:978-82.
6. Brent D y col. Risk factors for adolescent suicide: a comparison of adolescent suicide victims with suicidal inpatients. Arch Gen Psychiatry 1988;45:581-8.

7. Martin A y col. Age effects on antidepressant - induced manic conversion. Arch Pediatr Adolesc Med. 2004;158:773-780. 